\title{
Intraoperative Mapping and Radiofrequency Ablation of the His Bundle in a Patient with Complex Congenital Heart Disease and Intractable Atrial Arrhythmias Following the Fontan Operation
}

\author{
GONZALO URCELAY, MACDONALD DICK II, EDWARD L. BOVE, \\ RALPH MOSCA, PARVIN C. DOROSTKAR, GERALD A. SERWER, \\ ROBERT M. CAMPBELL, and BRIAN ARMSTRONG \\ From the Divisions of Pediatric Cardiology and Thoracic Surgery, C.S. Mott Children's Hospital, \\ the Departments of Pediatrics and Surgery, University of Michigan Ann Arbor, Michigan, and \\ The Children's Heart Center, Egleston Hospital, Emory University, Atlanta, Georgia
}

URCELAY, G., ET AL.: Intraoperative Mapping and Radiofrequency Ablation of the His Bundle in a Patient with Complex Congenital Heart Disease and Intractable Atrial Arrhythmias Following the Fontan Operation. Intraoperative mapping and radiofrequency ablation of the His bundle (with epicardial ventricular pacing) were performed in an 18-year-old woman with complex congenital heart disease and intractable chronic atrial arrhythmias following the Fontan operation. The presence of complex intracardiac anatomy as well as the exclusion of the atrioventricular conduction tissue from the systemic venous circulation strongly influenced the technical approach. (PACE, Vol. 16, July 1993, Part I)

atrioventricular conduction, congenital heart disease, Fontan operation, radiofrequency ablation, supraventricular tachycardia

\section{Introduction}

Postoperative supraventricular tachyarrhythmias are common complications of the Fontan operation. Atrial flutter as well as other forms of atrial reentry tachycardia have been described. ${ }^{1-4}$ Treatment, including antiarrhythmic agents and antitachycardia pacing devices, have met with variable success. ${ }^{4}$ Permanent interruption of atrioventricular $(\mathrm{AV})$ conduction by electrical or surgical ablation may be the only method available to ameliorate the symptoms associated with the rapid ventricular response that can accompany the atrial arrhythmias. ${ }^{5-9}$ In this report we present a patient with complex congenital heart disease, who, following the Fontan operation, developed

Address for reprints: Macdonald Dick II, M.D., Box 0204 F1310, C.S. Mott Children's Hospital, University of Michigan, Ann Arbor, MI 48109. Fax: (313) 936-9470.

Received December 16, 1992; revision February 1, 1993; accepted February 2, 1993. intractable chronic symptomatic atrial arrhythmias. His-bundle mapping and radiofrequency ablation (RFA) was accomplished in the operating room.

\section{Case Report}

An 18-year-old woman with an unbalanced AV septal defect (AVSD), large inlet ventricular septal defect, small left ventricle, double outlet right ventricle, underwent banding of the pulmonary artery at 9 months of age. At 5 years 6 months of age she underwent a Fontan operation involving suture closure of a secundum atrial septal defect and right atrial appendage to main pulmonary artery (MPA) anastomosis. The MPA was oversewn at the site of the previous band, and a patch was sewn into the right atrium so as to exclude the $\mathrm{AV}$ valve and coronary sinus (CS) from the caval return. Chronic recurrent atrial tachycardias in the form of intraatrial reentry and atrial flutter, requir- 
ing multiple hospitalizations for transthoracic DC cardioversion, complicated her late postoperative course. Antiarrhythmic drugs (including: digoxin, quinidine, digoxin and flecainide, and digoxin and amiodarone) were administered without consistent improvement. At 16 years 9 months of age a transvenous antitachycardia pacemaker (Intertach II, Intermedics, Inc., Angleton, TX, USA) system was placed in the physiological right atrium. Despite satisfactory function of the pacemaker along with a number of different drug combinations, she continued to have frequent symptomatic episodes of tachycardia with a rapid ventricular response. It was decided to attempt ablation of AV conduction.

At age 18 years and 9 months, the patient underwent electrophysiological study. A 7 French Mansfield electrode catheter (Boston Scientific, Watertown, MA, USA) was passed retrogradely from the aorta to the systemic ventricle and into the pulmonary venous atrium. Failure to obtain a His-bundle electrogram resulted in unsuccessful RFA (Radionics generator RFG-3C, Burlington, MA, USA) of the AV conduction tissue. Five days later the patient underwent operation. After establishing cardiopulmonary bypass, mapping of the AV node was attempted on the atrial side of the AV valve (AVV) ring near the CS; however, no Hisbundle electrogram could be recorded. Cryoablation was attempted, with transient 2:1 AV block observed during the tenth trial. Due to the prolonged pump time, the procedure was terminated and a DDD (Relay, Intermedics, Inc.) pacemaker was implanted.

Due to the failure of the first two procedures to improve the patient's vulnerability to her severely symptomatic and life limiting arrhythmias, reoperation was performed 5 days later. After institution of extracorporeal circulation, the pulmonary venous atrium was opened by removing the previously placed patch. Using a handheld 7 French Mansfield quadripolar catheter, with a 4-mm electrode tip, mapping was performed in the region of the triangle of Koch, the AV valve ring, and the CS; however, a His-bundle electrogram could not be recorded. Nonetheless, because of the presumed proximity of the underlying AV conduction tissue, multiple (unsuccessful) trials of RFA were delivered in this area. The catheter was then passed into the ventricle and its tip positioned in the AV groove underneath the posterior leaflet of the common AVV. Manipulation of the catheter tip by the exploring finger inserted through the $A V$ valve into the ventricle produced transient $A V$ block. To place more precisely the catheter's electrode tip against the AV groove, the posterior leaflet was incised $2-3 \mathrm{~mm}$ from the annulus, and the catheter passed beneath it against the AV groove, yielding a His-bundle electrogram (Fig. 1). Radiofrequency energy delivered at this site produced complete heart block (Fig. 2). The AV valve was repaired and the operation completed. The patient was discharged from the hospital 2 weeks later, with complete heart block and a normally functioning DDD pacemaker.

\section{Discussion}

This report underscores several new, critical considerations in the management of intractable atrial arrhythmias in the post-Fontan patient. First, transvenous access to the AV node and His bundle may be difficult to achieve because these structures and their radiological landmarks are often excluded from the systemic venous return. Although intraoperative mapping in patients with AV septal (AV canal) defects is useful in delineating the AV conduction tissue,${ }^{10}$ we were unable to detect in this patient the His-bundle potential mapping from the atrial side of the AV groove, most likely due to the complex AV anatomy and the displaced AV conduction system away from the usual anatomical landmark at the apex of the triangle of Koch. ${ }^{11}$ This failure to record the Hisbundle potential resulted in unsuccessful cryoablation and RFA within the atrium. Second, transvenous pacing of the ventricle following creation of complete heart block is not feasible in the patient following the Fontan operation for two reasons: the single systemic ventricle cannot be accessed from the systemic venous circulation, and, because of the risk of arterial emboli developing from an arterial endocardial pacing lead, an epicardial lead system is required. Third, the cryoablation technique required two different probes, one for mapping and one for ablation, effectively dissociating the mapping and ablating procedure, and thus adding a step that could introduce error in the exact placement of the ablating catheter. The use of the catheter with the electrode at the tip for mapping and ablation eliminates this potential 


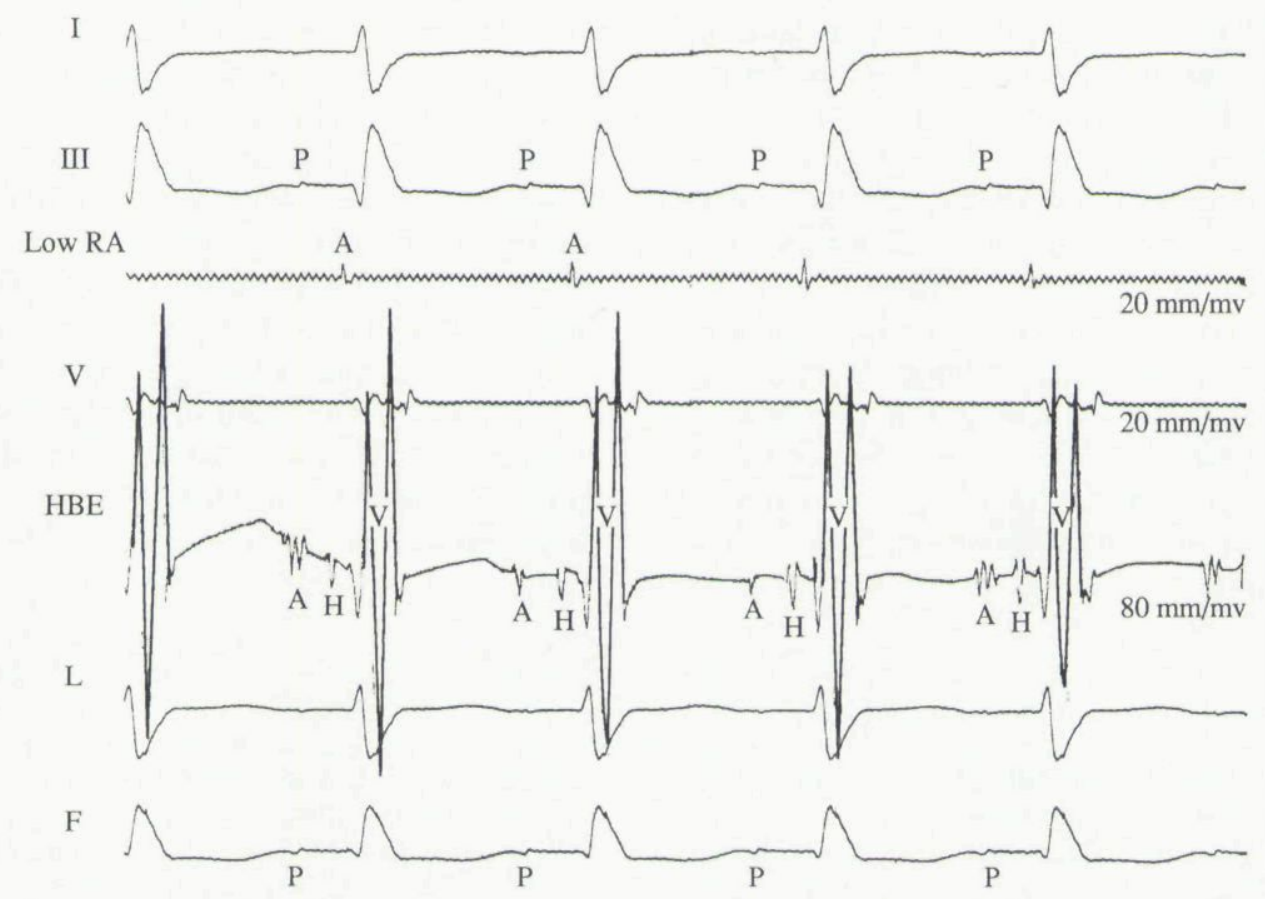

Figure 1. This figure portrays four surface electrocardiographic leads and two electrograms during mapping of the His bundle through the AV valve. Note the low amplitude P waves seen following extensive atrial surgery such as the Fontan operation. The a wave reflects activation low in the atrium. $\mathrm{H}$ denotes the His-bundle potential. The tracings have been redrawn for clarity. $A=$ low atrial electrogram; $F=A V F$ electrocardiographic lead; $H=$ His-bundle electrogram; $\mathrm{HBE}=$ His-bundle electrogram tracing; $L=A V L$ electrocardiographic lead; Low RA = low right atrium electrogram tracing; $I=I$ electrocardiographic lead; $I I I=I I I$ electrocardiographic lead; and $V=$ ventricular electrogram tracing.

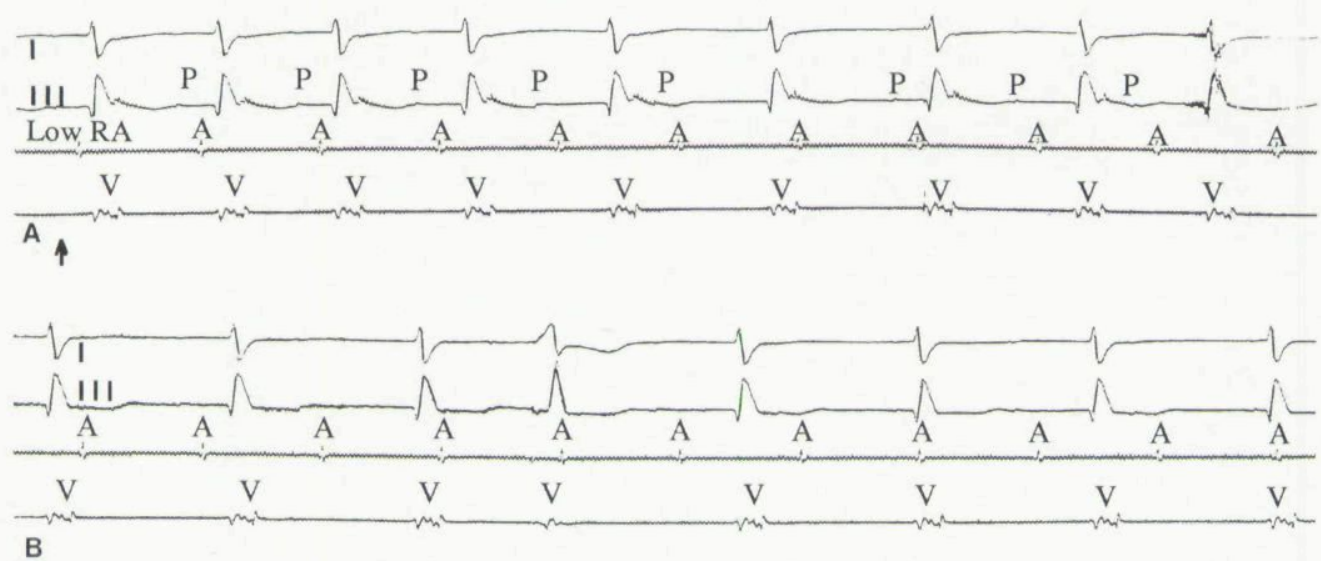

Figure 2. This tracing is recorded during delivery of radiofreqency energy (onset at arrow) between an indifferent lead placed over the left scapula on the back and the same distal electrode used to map the His bundle (Fig. 1). Panels A and B are continuous. Note the development of $A V$ block (fourth $P$ wave) progressing to complete heart block. The tracings have been redrawn for clarity. $V$ = ventricular electrogram. All other abbreviations are the same as in Figure 1. 
problem. Finally, the His bundle itself, rather than the AV node, may be the more suitable target for mapping and ablating AV conduction in the patient with anatomical defects in the region of the AV canal. Although in our patient this target was reached through the AV valve at surgery, it is quite conceivable that the intraventricular bifurcating portion of the His bundle could be mapped and ablated from a transvenous retrograde aortic to ventricular approach exploring the AV groove with the electrode tip, rather than crossing retrogradely the AV valve and attempting (as we unsuccessfully did) to map and ablate the penetrating

\section{References}

1. Chen S, Nouri S, Pennignton DG. Dysrhythmias after modified Fontan procedure. Pediatr Cardiol 1988; 9:215-219.

2. Weber HS, Hellenbrand WE, Kleinman CS, et al. Predictors of rhythm disturbances and subsequent morbidity after the Fontan operation. Am J Cardiol 1989; 64:762-767.

3. Leung MP, Benson LN, Smallhorn JF, et al. Abnormal cardiac signs after Fontan type of operation: Indicators of residua and sequelae. Br Heart J 1989; 61:52-58.

4. Kürer CC, Vetter VL. Electrophysiology after Fontan repair of tricuspid atresia. In PS Rao (ed.): Tricuspid Atresia. Mt. Kisco, NY, Futura Publishing Company, Inc., 1992, pp. 387-400.

5. Scheinman MM, Morady F, Hess DS, et al. Catheter-induced ablation of the atrioventricular junction to control refractory supraventricular arrhythmias. J Am Med Assoc 1982; 248:851-855.

6. Gallagher JJ, Svenson RH, Kasell JH, et al. Catheter technique for closed-chest ablation of the atrioventricular conduction system: A therapeutic alternative for the treatment of refractory supraventricular tachycardia. N Engl J Med 1982; 306:194-200. portion of the His bundle. Nonetheless, even with this approach, operation would be necessary to implant the epicardial pacing system.

In summary, intraoperative mapping and RFA of the His bundle (with postablation ventricular pacing) represents a definitive method of achieving a controlled rhythm in patients with intractable chronic atrial arrhythmias complicating the Fontan operation. In these patients, the presence of complex intracardiac anatomy as well as the exclusion of the target tissue from the systemic venous circulation strongly influences the technical approach.

7. Huang SK, Bharati S, Graham AR, et al. Closedchest catheter desiccation of the atrioventricular junction using radiofrequency energy - a new method of catheter ablation. J Am Coll Cardiol 1987; 9:349-358.

8. Scheinman MM, Laks MM, DiMarco J, et al. Current role of catheter ablative procedures in patients with cardiac arrhythmias: A report for health professionals from the Subcommittee on Electrocardiography and Electrophysiology, American Heart Association. Circulation 1991; 83: 2146-2153.

9. Yeung-Lai-Wah JA, Alison JF, Lonergan L, et al. High success rate of atrioventricular node ablation with radiofrequency energy. J Am Coll Cardiol 1991; 18:1753-1758.

10. Dick M, Norwood WI, Chipman C, et al. Intraoperative recording of specialized atrioventricular conduction tissue electrograms in 47 patients. Circulation 1979; 59:150-160.

11. Wilcox BR, Anderson RH. Surgical Anatomy of the Heart. New York, NY, Raven Press, 1985, pp. 6-16. 
This document is a scanned copy of a printed document. No warranty is given about the accuracy of the copy. Users should refer to the original published version of the material. 